\title{
Coarctation of the aorta and post-stenotic aneurysm formation
}

\author{
I M Mitchell, J C S Pollock
}

\begin{abstract}
Despite earlier angiography, poststenotic aneurysm of the aorta was an unexpected finding at operation in two patients with coarctation. One aneurysm was found in an intercostal artery in a 19 year old man and the other was a false aneurysm just distal to the coarctation site in a 7 year old boy. These aneurysms are fragile, apt to rupture, and difficult to diagnose preoperatively. Though local factors such as jet streams and bacterial endocarditis may influence their formation there must be an underlying generalised weakness in the arterial wall.

A coarctation should not be regarded as an isolated arterial abnormality because it may be a feature of a more generalised disease. Because of the risk of rupture, which may not be prevented by antihypertensive treatment, operation should not be delayed in any age group.
\end{abstract}

Coarctation of the aorta is one of the most common congenital cardiac abnormalities, occurring in about 1 in 1200 live births. In untreated patients the average life expectancy (from one series of 200) was 32 years, ${ }^{1}$ with the cause of death (according to Campbell ${ }^{2}$ ) being cardiac failure $(25.5 \%)$, endocarditis $(18 \%)$, cerebrovascular haemorrhages $(11.5 \%)$, "aortic" rupture (21\%), and unknown reasons $(24 \%)$.

The association of aneurysms with coarctation of the aorta is well recognised. In a series of 1091 patients reviewed in 1960, Skandalakis et al reported 102 aneurysms of which 63 were distal to the coarctation with 12 in the intercostal arteries. ${ }^{3}$ It is important to detect such aneurysms because rupture was the cause of death in $76 \%$ of patients with aneurysms. Although computed tomography may be helpful, angiography remains the mainstay of diagnosis, yet even with this, aneurysms are often discovered only at operation. Though the development of aneurysms proximal to the coarctation might be expected (because of hypertension in the upper part of the body), aneurysms are equally common distal to the coarctation site and are just as likely to rupture. We present two such cases and discuss the possible mechanisms leading to aneurysm formation.

\section{Case reports}

CASE 1

A 19 year old man was referred to us in April
1989 after a routine medical examination when he was found to have hypertension with a blood pressure of $170 / 110 \mathrm{~mm} \mathrm{Hg}$ in the right arm, no femoral pulses, and a systolic bruit. On questioning he complained of a reduced exercise tolerance, claudication in both legs, and slight breathlessness on exertion. An electrocardiogram showed sinus rhythm with evidence of minimal left ventricular hypertrophy. Echocardiography and angiography confirmed left ventricular hypertrophy with good left ventricular function, but also a coarctation of the aorta with normal distal arteries.

Treatment with atenolol was started while he awaited operation. At operation a postductal coarctation was found. This was resected and a gelatin impregnated knitted Dacron graft (Vascutek, Inchinnan, Scotland) was sutured into place to restore aortic continuity. In addition to the coarctation, however, a $1 \times 1 \mathrm{~cm}$ aneurysm of the first left intercostal artery was found. This was very thin walled and at risk of rupture, with the dilated portion starting $0.5 \mathrm{~cm}$ away from an aortic ostium of normal size (figure). The intercostal artery was transected proximally and ligated distally and the aortic ostium oversewn. He made a good recovery postoperatively and was well at follow up 7 months later though treatment with atenolol was continued.

\section{CASE 2}

A 7 year old boy presented to us in 1983 with a history of claudication in both legs when running and of becoming easily fatigued. On examination he was found to be hypertensive with a blood pressure of $150 / 90 \mathrm{~mm} \mathrm{Hg}$ in the right arm, no femoral pulses, and a systolic murmur at the left sternal edge. An electrocardiogram was unremarkable but angiography showed a coarctation of the aorta and mild aortic incompetence. At operation the coarctation was confirmed and was repaired

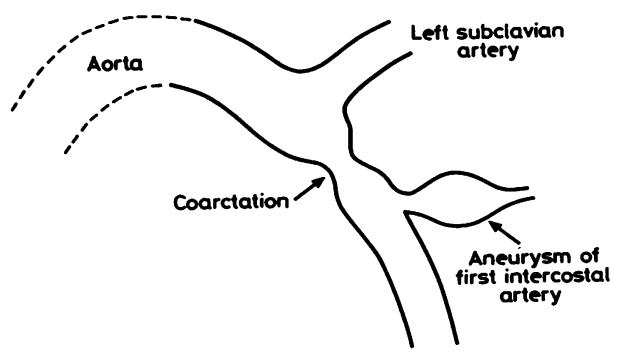

Drawing showing coarctation of the aorta and the aneurysm of the first intercostal artery in case 1 . 
by widening the aorta with a diamond shaped Dacron patch. A $2 \mathrm{~cm}$ false aneurysm of the aorta was also found $3 \mathrm{~cm}$ distal to the coarctation. Over most of the circumference the wall consisted only of adventitia, and the aneurysm was repaired with Teflon buttressed sutures and reinforced by a Teflon collar around the aorta with an internal diameter of $2 \cdot 5-3 \mathrm{~cm}$. Postoperative recovery was uneventful and 6 years later he remained well, normotensive, and on no medication.

\section{Discussion}

Coarctation of the aorta is usually repaired before the age of 5 years because of the increasing risk of irreversible hypertension and left ventricular hypertrophy developing when repair is delayed. An equally serious and potentially fatal complication of a coarctation, but one that is less well appreciated, is aneurysm formation. Aneurysms may be single or multiple and may occur in the aorta, intercostal arteries, Abbott's artery, or the cerebral vessels. Apart from the risk of rupture (that may lead to sudden death) or a cerebrovascular accident, pressure on the anterior spinal artery or the spinal cord from an intercostal artery aneurysm can also lead to paralysis. ${ }^{5}$

The existence of so many arterial abnormalities suggests that a coarctation may be a feature of a more generalised arterial disease rather than a discrete entity. Bicuspid aortic valves are, for example, a frequent association. ${ }^{1}$

The cause of aneurysm formation is unclear but in the upper part of the body hypertension is a possibility. There is some evidence that coarctectomy does not protect against hypertension if the operation is delayed beyond the first few years of life and hypertension has been uncontrolled. ${ }^{4}$ None the less, the pressure required to produce medial dissection is likely to be far greater than aortic blood pressure, even in cases of severe hypertension. ${ }^{67}$ This implies that for an aneurysm to develop there must also be some congenital weakness in the arterial wall. The finding that only $32 \%$ aneurysms were proximal to the coarctation in one large series ${ }^{3}$ is further evidence against the role of hypertension.

In our two cases aneurysms developed on the "low flow" side of the coarctation and therefore hypertension was clearly not the cause. A role has been suggested for bacterial endocarditis, ${ }^{8}$ which is undoubtedly important in some cases, perhaps aided by eddy currents in low flow areas. One further possibility is that some poststenotic aneurysms may be directly attributable to jet streams of blood coming through the narrowed segment and impinging on the distal aortic wall. Skandalakis et al suggested that this might have accounted for $6 \%$ of coarctations in their series; ${ }^{3}$ it was probably the cause of the false aneurysm in our patient 2 . Nevertheless, a congenital weakness in the structure or biochemistry of the artery wall may well account for most cases. When aneurysms arise in the region of the ligamentum arteriosum it is possible that ductal tissue could become incorporated into the aortic wall during development, so as to produce a local weak spot. The more widespread appearance of aneurysms may be explained by the evidence that there is destruction of elastic tissue in the tunica media of the arterial wall together with an alteration in the mucopolysaccharide protein matrix. ${ }^{7}$ With this background, hypertension or poststenotic jet streams may then be compounding factors. In case 1 , however, the intercostal aneurysm was on the low flow side of the coarctation and arose some distance away from the aorta and the coarctation site; so it is unlikely that either factor was operating. In contrast, the false aneurysm in case 2 may well have been caused by abnormal stress on the aortic wall from a jet stream. Certainly it is unusual for an aneurysm to develop in such a young patient.

There is no doubt that there is a strong association between coarctation of the aorta and aneurysm formation. Though to date there has only been one definite report of rupture of an aneurysm of an intercostal artery ${ }^{9}$ (perhaps because these are misreported as aortic ruptures) there is no doubt that aneurysms distal to the coarctation are common and are potential sites of rupture. Both our patients underwent preoperative angiography but this did not detect the aneurysms, both of which were about to rupture. These fragile, thin walled lesions are often found unexpectedly at operation, and they do not tolerate manipulation during coarctation repair. ${ }^{49}$ Once detected, coarctation of the aorta should be operated upon without delay - in adults and older children just as in neonates-because of the potentially fatal consequences of rupture of an unsuspected aneurysm. It is unwise to maintain such patients on antihypertensive treatment and defer operation. Though thoracic aneurysms will be detected and can be dealt with at the time of coarctation repair, associated cerebral aneurysms are likely to be clinically silent and will remain undetected. Because $30-50 \%$ of patients operated on in late childhood or adulthood remain hypertensive after operation 4 and because coarctectomy itself will not protect against rupture, it is important to provide long term antihypertensive treatment. ${ }^{10}$

1 Abbott ME. Coarctation of the aorta of the adult type. 11. A statistical study and historical retrospect of 200 recorded cases with autopsy, of stenosis or obliteration of the descending arch in subjects above the age of two years. Am Heart J 1928;3:574-618.

2 Campbell $M$. Natural history of coarctation of the aorta. $B$ Heart $J$ 1970;32:633-40.

3 Skandalakis JE, Edwards BF, Gray SW, Davis BM, Hopkins WA. Coarctation of the aorta with aneurysm. Surg ins WA. Coarctation of the aorta with aneurys

4 Westaby S, Parnell B, Pridie RB. Coarctation of the aorta in Westaby S, Parnell B, Pridie RB. Coarctation of the aorta in
adults. Clinical presentation and results of surgery. $J$ adults. Clinical presentation and

5 Brewer LA, Fosburg RG, Mulder GA, Verska JJ. Spinal cord complications following surgery for coarctation of the aorta. A study of 66 cases. J Thorac Cardiovasc Surg 1972;64:368-81.

6 Robertson JS, Smith KV. An analysis of certain factors associated with production of experimental dissection in aortic media in relation to pathogenesis of dissectin aneurysms. J Path Bact 1948;60:43-9.

7 Burman SO. Medial degeneration and its relation to dissecting aneurysm. Surg Gynecol Obstet 1960;110:Int Abst Surg 1-8.

8 Sellors TH. Coarctation of the aorta associated with aneurysm treated by aortic resection and grafting. $\mathrm{Br}$ Surg 1956;43:365-71.

9 Wallace RB, Nast EP. Postcoarctation mycotic intercosta arterial pseudoaneurysm. Am J Cardiol 1987;59:1014-5.

10 Hodes HL, Steinfeld L, Blumenthal S. Congenital cerebral aneurysms and coarctation of the aorta. Arch Pediat 1959;76:28-43. 